\title{
A Novel Algorithm for Efficient Channel Estimation using MMSE-Kalman Estimator and Median Filtering
}

\author{
Naincy Valdaya \\ M-Tech Research Scholar \\ Department of ECE \\ NRI Institute of Research and \\ Technology, Bhopal, \\ Bhopal
}

\author{
Mukesh Kumawat \\ Research Guide \\ NRI Institute of Research and \\ Technology, Bhopal, \\ Bhopal
}

\author{
Sachin Murarka \\ Co-Guide \\ Department of ECE \\ NRI Institute of Research and \\ Technology, Bhopal, \\ Bhopal
}

\begin{abstract}
Wireless communication is a emerging field of evolution in modern era of technology, and every day it is giving new technology to facilitate human being. The new facilities always need a QoS i.e. directly user satisfaction. For satisfaction of wireless users is to give efficient and reliable technology, so that sharing of information is made easier. In the same context the channel of wireless communication system need extra attention for efficient delivery of information from one place to another wirelessly. In this paper we are trying to estimate the channel using MMSE-Kalman Estimator in OFDM system with median filtering to enhance the performance of the system. The proposed methodology is implemented and figure of merits are calculated as BER and MSE, and we have found that the use of median filter with MMSE-Kalman estimator is good.
\end{abstract}

\section{Keyword}

MMSE, Kalman Estimator, Median Filter, BER and MSE

\section{INTRODUCTION}

A central issue in the wireless communications setting is the signal fading and inter symbol interference (ISI) introduced by the channel [1]. Due to multipath propagation, many copies of the transmitted signal arrive at the receiver antenna, every with a given attenuation and delay. When these delays are separated by more than the symbol duration, inter symbol interference (ISI) results. Whereas, when the receiver antenna is set in motion, as is usually the case in such applications such as cellular telephony, signal fading results as the received power level fluctuates because the multipath components add constructively or destructively. Therefore as a result of these distortions, wireless fading channels show dramatically poorer bit-error performance than traditional additive white Gaussian noise channels when using uncoded transmissions.

The idea behind this sort of precoding is to temporally distribute the energy of each and every symbol, and effectively recombine these transmissions, to achieve the average effect of the channel rather than the instantaneous fade. A key element of the receiver is an equalizer that partially compensates for the instantaneous ISI and fading effects of the channel, and constructing the equalizer requires some information of the fading channel impulse response. In a real wireless system, particularly when the receiver is mobile, information of the fading characteristics must be obtained through adaptive channel estimation, either blind or trained, taking into account a model for the channel. The impact of estimation errors must then be incorporated into the performance analysis of the communication system. Various techniques for estimating the channel must be compared based on their bit-error rate probability and computational complexity.

Types of Fading:
The propagation model mainly focuses on predicting the average received signal strength at a given T-R (Transmitter-Receiver) separation and radial variation for the specified separation. So we can classify fading into two types: Large-scale fading and Small scale fading. Large scale fading attributes for variation in signal strength over large T-R separation distances. Large scale models try to find out mean signal power attenuation or path loss due motion over large area around transmitter or receiver. Small scale fading characterizes rapid fluctuation of received signal strength over short T-R separations and for short period of time. So the signal is a sum of many signals coming from different directions with different attenuation which brings dramatic changes in signal amplitude and phase.

Various models exist in literature for large scale fading. They are like empirical models such as Okumura model, Hata model, cost 136 model etc; indoor models like Log-distance path loss model, Ericsson numerous breakpoint model, Attenuation factor model etc. Large scale fading models find applications in wireless network planning for an area and modeling path loss over a large distance. So, large scale path loss models are more important for cell site planning but less for communication system design. So we will next discuss small scale fading in a little detail.

Types of Small-scale Fading:

Small-scale fading occurs due to two propagation mechanisms as described above [12]. They are

Due to multipath delay spread

- $\quad$ Flat fading

- $\quad$ Frequency selective fading

Due to Doppler spread

- $\quad$ Fast fading

- Slow fading

If the bandwidth of the channel is less than range of frequency over which the channel has constant gain and linear phase, then the signal undergoes flat fading. This type of fading is common in literature as this is analogous to a low pass filter. After passing the spectral characteristics of the channel remains unchanged but the gain changes with time.

\section{ESTIMATING THE CHANNEL}

The mobile radio channel sharply influences the performance of wireless communication system and needs rigorous analysis before modeling any system. The transmission path between the transmitter and the receiver can be a simple line of sight to a severely faded by trees, buildings and mountains. Radio channels are random and time varying in nature, so don ${ }^{\text {et }}$ offer a 
smooth analysis. So modeling the radio channel taking all the influencing parameters into account is a tough task. So estimating the channel by using its response to a known data and repeating this process time and again, there by using channel statistics. Various estimation techniques are used in literature, here we have exploited the use of LS, MMSE and Modified MMSE estimator.

\section{Minimum Mean Square Error (MMSE) Estimation:}

If the $\mathrm{g}$ is uncorrelated with $\mathrm{W}$ then the time domain MMSE estimator is given by [22]

$$
\begin{aligned}
& \hat{\mathrm{g}}_{\mathrm{MMSE}}=\mathrm{R}_{\mathrm{Yg}} \mathrm{R}_{\mathrm{YY}}{ }^{-1} \mathrm{Y} \\
& \text { where }
\end{aligned}
$$

$\mathrm{RYg}$ : cross-covariance matrix of $\mathrm{Y}$ and $\mathrm{g}$

RYY : auto-covariance matrix of Y

$\hat{\mathrm{h}}_{\mathrm{MMSE}}=\mathrm{F} \hat{\mathrm{g}}_{\mathrm{MMSE}}$

For MMSE estimator is given by

$$
\begin{aligned}
& \mathrm{Q}_{\mathrm{MMSE}}=\mathrm{R}_{\mathrm{gg}}\left[\left(\mathrm{F}^{\mathrm{H}} \mathrm{X}^{\mathrm{H}} \mathrm{XF}\right)^{-1} \sigma_{\mathrm{n}}{ }^{2}+\mathrm{R}_{\mathrm{gg}}\right]^{-1}\left(\mathrm{~F}^{\mathrm{H}} \mathrm{X}^{\mathrm{H}} \mathrm{XF}\right)^{-1} \\
& \mathrm{R}_{\mathrm{gg}}=\text { auto-covariance matrix of } \mathrm{g}
\end{aligned}
$$

Kalman Filter for Channel Estimation:

The Kalman filter is an efficient recursive algorithm which estimates the state of a dynamic system from a series of noisy measurements [25, 26]. Kalman filter has been applied in communication systems since 1970s [18-19]. Lawrence and Kaufman [18] first applied Kalman filter in the equalization of digital communication systems. The proposed algorithmic [18] do not exploit any prior information about the channel state, and it can only be applied in time invariant channel. The state equation is made based on the information symbol, with the driving noise as the information symbol. The state equation said nothing more than that each succeeding component at time $\mathrm{i}$ is equal to the previous component at time $\mathrm{i}$ with known channel state, the state variables shall be estimated based on the observation. When the channel state is not known, the state model needs to be extended in which all the unknown parameter, the adding information symbol and the channel state, are included.

Kalman filter could also be applied to track the states of time varying channels [21-24]. The state variable is defined as the channel states, which can be modeled in an AR model [21]. The state equation is then built based on the AR model, which reflects the statistics of time varying channel. Iltis [21] proposed a Kalman filter to track multi-path channel in a direct-sequence, the spread-spectrum communication systems. Iltis and Fuxjaegera $[21,22]$ modeled the time-varying tap coefficients as autoregressive (AR) processes with respect to time, and then employed an extended Kalman filter to adaptively estimate them. Their methods take advantage of the channel's time domain statistics, but no method is provided to estimate the AR model parameters. Additionally, the assumption of uncorrelated tap coefficients further limits the applicability of the method.

The contribution of this work is a Kalman filter based framework of channel equalizer in a time-frequency-fading environment. The gains of the sub-channels are defined as the state variables, and an AR process is used to model the dynamics of the channel. Then Kalman filter could be applied to estimate the channel from the received OFDM signals when pilot symbols are available. This Kalman filter is of a dimension equal to the product of the number of sub-carriers and the order of the AR model, which can be very large. To reduce its complexity, persubcarrier Kalman filter scheme is proposed, which is the Kalman filter channel estimator is applied to obtain the gain of each sub-carrier independently, and then a minimum meansquare-error (MMSE) combiner is used to refine the estimates. The per-subcarrier Kalman estimator investigates the timedomain correlation of the channel, while the MMSE combiner investigates the frequency domain correlation. This two-step solution provides a performance comparable to the much more complicated Kalman joint estimator.

The Kalman filter method is also extended to give a blind channel estimation algorithm based on the mixture Kalman filter (MKF). The MKF uses Monte Carlo simulations to do filtering. By simulating the transmitted symbol sequences according to their a posteriori probabilities, so this is called importance sampling, and feeding them to the Kalman filter channel estimator, a Bayesian estimate of the channel can be obtained through averaging the Kalman filter output of every simulation. The MKF technique applies to the joint estimation of all subcarriers and also works in the per-subcarrier fashion with a much reduced complexity. In addition to the channel estimate, the MKF could directly give symbol detection.

\section{PROPOSED METHODOLOGY}

Wireless communication is needed to reduce the effects of error introduced by the wireless channel by changing its nature varies frequently. For this there should be a method to detect and estimate the channel characteristics so that system can change the nature of it to compensate the noises. In this section the proposed methodology is explained in detail. The Block diagram and flow chart of the proposed methodology is given in below figures.

The block diagram shows the Proposed Methodology for wireless system with QAM-64 modulation and Median Filtration Methodology in Fig. In the proposed methodology QAM-64 modulation with Median Filter has been used to minimize the Bit Error Rate is calculated with SNR. In the transmitter section firstly the data is modulated by 64-QAM modulator and then Pilot Insertion is done after this IFFT is applied for multiplexing then after addition of cyclic prefix is done with data signal through the Rayleigh Fading Channel the noise is mixed. In the receiver section then cyclic prefix is removed FFT is applied for de-multiplexing then Pilot Removal \& Demodulator has been used then after MMSE Kalman Estimator and Median Filter is adopted to reduce the BER. 


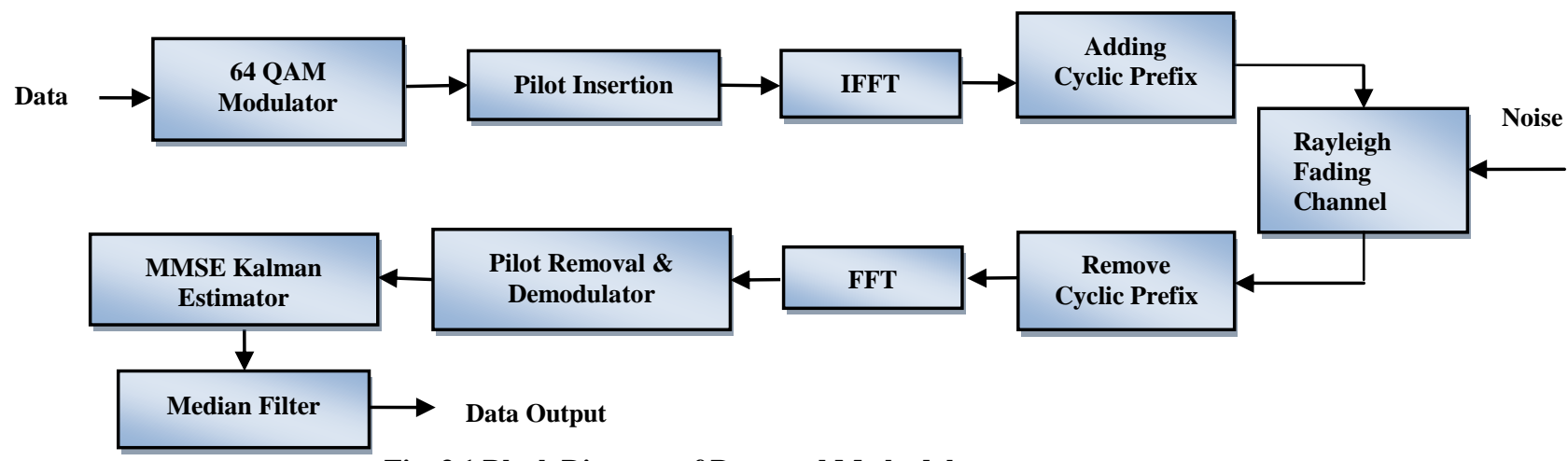

Fig. 3.1 Block Diagram of Proposed Methodology

As the above flow graph shows the simulation flow, in this the environmental variables are initialized the then signal is generated, 64-QAM Modulator is adopted after that OFDM Pilot Insertion is done after a OFDM the signal is transmitted through Rayleigh Fading Channel \& Adding Noise then OFDM DEMUX Technique is used after that the MMSE with Kalman Estimation is implemented then 64- QAM modulation and then Median Filter is adopted for minimizing the BER.

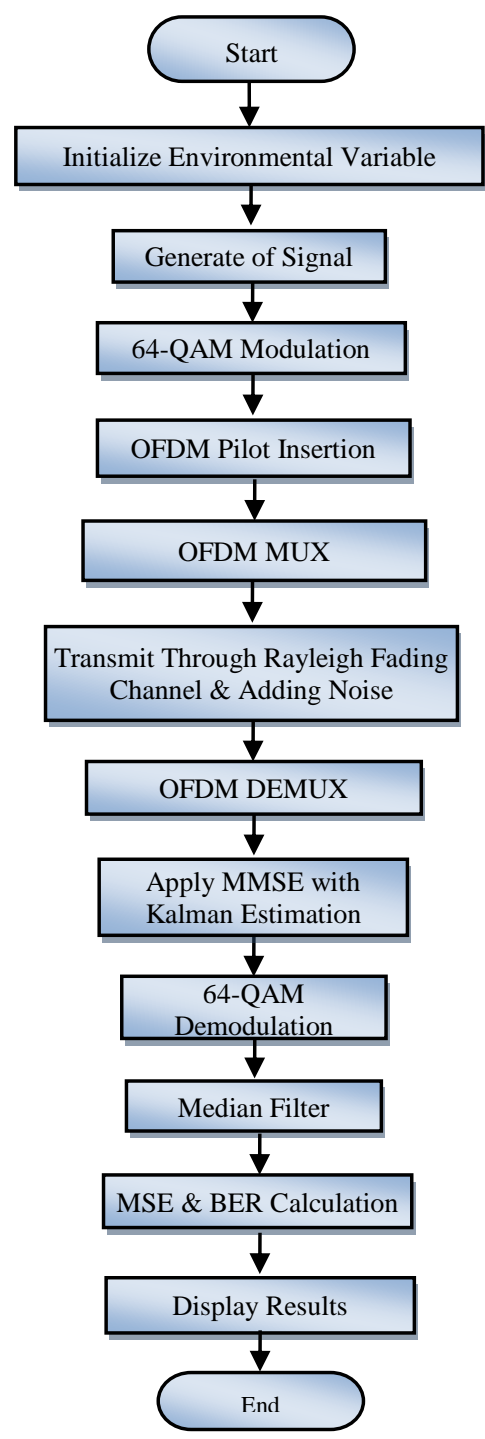

\section{SIMULATION RESULTS}

In wireless communication channel estimation is the need to enhance the reliability and performance of the system. Here we have calculated the two parameters bit error rate(BER) and mean square error(MSE). In Fig. 4.1 BER performance of proposed methodology is shown for MMSE Kalman Estimator with and without filter.

BER vs SNR curve shows the performance of the system as the value of BER reduces the performance of the system increases. It can be said that in other aspects that of the value of BER is for lower values of SNR that means the systems is good even on the longer distances. In Fig. 4.1 we have calculated the values of BER vs SNR for MMSE-Kalman Estimator with Median Filter and without Median Filter and from the results it is clear that the values(i.e. performance of the system is better for MMSEKalman Estimator with median filter.

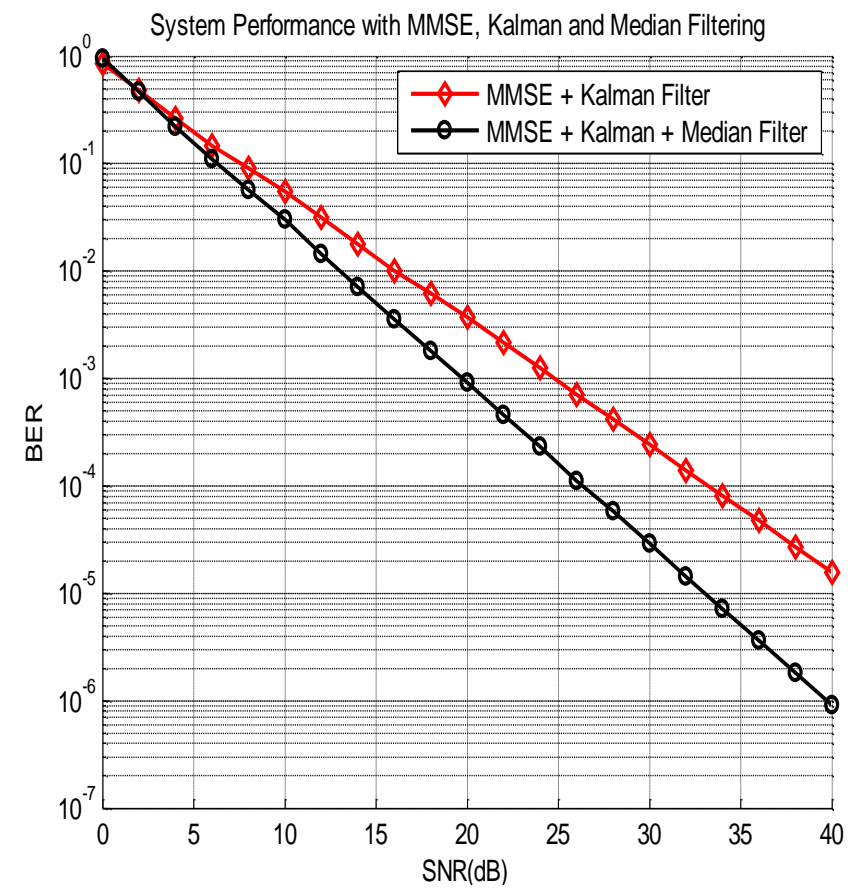

Fig. 4.1 BER performance of OFDM system using MMSEKalman Estimator and Median Filter

Fig. 3.2 Flow Chart of Proposed Methodology 


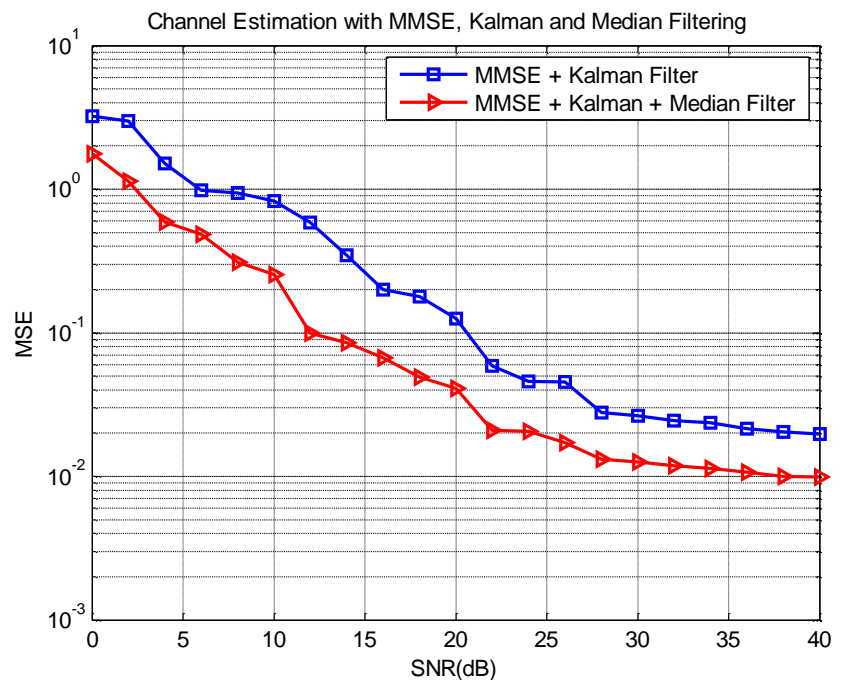

Fig. 4.2 MSE performance of OFDM system using MMSEKalman Estimator and Median Filter

MSE vs SNR curve shows the average error of the system as the value of MSE reduces the average error produces by the channel is compensated. It can say that in other aspects that value of MSE is for lower values of SNR that means the system can sustain even on the longer distances. In Fig. 4.2 we have calculated the values of MSE vs SNR for MMSE-Kalman Estimator with Median Filter and without Median Filter and from the results it is clear that the values(i.e. average error of the system is better for MMSE-Kalman Estimator with median filter.

\section{CONCLUSION AND FUTURE SCOPE}

The proposed methodology is implemented and the result is extracted which is shown in previous section. Now for the proper estimation and better performance proposed methodology is giving better results than previous methods. From the simulation results we can say that the use of MMSE-Kalman Estimator followed by median filtering giving better than MMSE-Kalman Estimator alone. After studying the methodology and the outcomes i.e. BER and MSE, we can say that if we can use the better estimator with median filter we can achieve better results or instead of median filter.

\section{REFERENCES}

[1] F. Wang, A. Ghosh, "Mobile WiMAX Systems: Performance and Evolution", IEEE Communications Magazine, vol. 46, no.10, October 2008, pp.41-49.

[2] WiMAX Forum, "WiMAX System Evaluation Methodology", Version 2.1, July 2008.

[3] R. Van Nee and R. Prasad, "OFDM for Wireless Multimedia Communications", Boston, Artech House, 2000.

[4] Daniel Larrson, "Analysis of channel estimation methods for OFDMA", Master of Science Thesis, Stockholm, Sweden 2006-12-19.

[5] Muhammad Saad Akram, "Pilot-based Channel Estimation in OFDM", Systems Master Thesis, Nokia Denmark, 2007.

[6] Sinem Coleri, Mustafa Ergen, Anuj Puri, and Ahmad Bahai,"Channel Estimation Techniques Based on Pilot Arrangement in OFDM Systems" IEEE transactions on broadcasting, vol. 48, no. 3, September 2002.
[7] Waiel Elsayed Osman, Tharek Abd. Rahman, "Optimization of Guard Time Length for Mobile WiMAX System over Multipath Channel”, IMECS 2008, Hong.

[8] Md. Zahid Hasan, "Comparative Study of Different Guard Time Intervals to Improve the BER Performance of Wimax Systems to Minimize the Effects of ISI and ICI under Adaptive Modulation Techniques over SUI-1 and AWGN Communication Channels", (IJCSIS) International Journal of Computer Science and Information Security, Vol. 6, No.2, 2009.

[9] ZHANG Zhao-yang and LAI Li-feng, "A novel OFDM transmission scheme with length-adaptive Cyclic Prefix", Journal of Zhejiang University SCIENCE ISSN 1009-3095, Nov. 2003.

[10] Greg Welch, Gary Bishop, "An Introduction to the Kalman Filter”, UNC-Chapel Hill, TR 95-041, July 24, 2006.

[11] M. Huang, X. Chen, L. Xiao, S. Zhou and J. Wang, "Kalman-filter- based channel estimation for orthogonal frequency-division multiplexing systems in time-varying channels", IET Communication, Vol. 1, No. 4, August 2007

[12] Hussein Hijazi, Amina Dakhlallah, Shafic Al Hajj, Ali Chamas Al Ghouwayel and Achraf Dhayni, “ Joint CFO and Time-varying Channel Estimation by Particle Filtering in OFDM Systems", The 3rd International Conference on Communications and Information Technology (ICCIT2013): Wireless Communications and Signal Processing, Beirut The 3rd International Conference on Communications and Information Technology (ICCIT2013) 2013 IEEE.

[13] Eric Pierre Simon and Mohammad Ali Khalighi, "Iterative Soft-Kalman Channel Estimation for Fast Time-Varying MIMO-OFDM Channels", IEEE Wireless Communications Letters, Vol. 2, No. 6, December 2013

[14] Bor-Sen Chen, Fellow, IEEE, Chang-Yi Yang, and Wei-Ji Liao "Robust Fast Time-Varying Multipath Fading Channel Estimation and Equalization for MIMO-OFDM Systems via a Fuzzy Method", IEEE Transactions On Vehicular Technology, Vol. 61, No. 4, May 20121599

[15] Muneer P. and S.M. Sameer, "Doubly-selective Channel Estimation for OFDM Systems Using Bernstein Basis Polynomials and Kalman-tracking" 2012 IEEE pp 603

[16] Ganesh Miriyala, Amit Kaul, Ravinder Nath , "Reduced Complexity Channel Estimation Method for Multi Input Multi Output-Orthogonal Frequency Division Multiplexing Systems by Subspace Tracking” 2012 IEEE pp 470.

[17] George Ignatius, Murali Krishna Varma U, Nitish. S. Krishna,Sachin. P.V , P.Sudheesh, "Extended Kalman Filter based Estimation for Fast Fading MIMO Channels" in 2011.

[18] R. E. Lawrence and H. Kaufman, "The Kalman filter for the equalization of a digital communications channel," IEEE Trans. Commun. Technol., vol. COMM-19, pp. 1137-1141, Dec. 1971

[19] D. Godard, "Channel equalisation using a Kalman filter for fast data transmission", IBM J. Res., pp. 267-273, Dec, 1974.

[20] Luvison and G. Pirani, "Design and performance of an adaptive Kalman receiver for synchronous data 
transmission,"IEEE Trans. Aerosp. Electron. Syst., vol. AES-15, pp. 635-648, September 1979.

[21] R. A. Iltis, "Joint estimation of PN code delay and multipath using extended Kalman filter," IEEE Trans. on Communications, vol. 38, no. 10, pp. 1677-1685, October 1990.

[22] R. A. Iltis, and A. W. Fuxjaeger, "A digital DS spreadspectrum receiver with joint channel and Doppler shift estimation," IEEE Trans. on Communications., vol. 39, no. 8, pp. 1255-1267, August 1991.

[23] M. K. Tsatsanis, G. B. Giannakis, and G. Zhou, "Estimation and equalization of fading channels with random coefficients," IEEE Acoustics, Speech, and Signal Processing, ICASSP-96. Conference Proceedings., Vol. 2, pp. 1093-1096, May 1996.

[24] G. T. Zhou, Yongsub Kim, G. B. Giannakis, "Estimation and equalization of time selective fading channels," Signals,
Systems, and Computers, 1999. Conference Record of the Thirty-Third Asilomar Conference on, Vol 1, pp. 248-252, Oct. 1999.

[25] R. E. Kalman, "A New Approach to Linear Filtering and Prediction Problems," Transactions of the ASME - Journal of Basic Engineering, Vol. 82, pp. 35-45, 1960

[26] R. E. Kalman, and R. S. Bucy, "New Results in Linear Filtering and Prediction Theory," Transactions of the ASME - Journal of Basic Engineering, Vol. 83, pp. 95-107, 1961

\section{AUTHOR'S PROFILE}

Naincy Valdaya is a research Scholar at NRI Institute of Science and Technology, under Rajiv Gandhi Proudyogiki Vishwavidyalaya, Bhopal. She is pursuing her Master of Technology in Digital Communication. She has keen interest in OFDM technology in wireless communication system. 